\title{
On the use of matrix-free shift-invert strategies for global flow instability analysis
}

\author{
Francisco Gómez ${ }^{a, *}$, José Miguel Pérez ${ }^{b}$, Hugh M. Blackburn ${ }^{a}$, Vassilis Theofilis ${ }^{b}$ \\ a Department of Mechanical and Aerospace Engineering, Monash University, Victoria 3800, Australia \\ ${ }^{\mathrm{b}}$ School of Aeronautics, Universidad Politécnica de Madrid, Plaza Cardenal Cisneros 3, E-28040 Madrid, Spain
}

Keywords:

Global linear instability analysis

Large-scale eigenvalue problems

Krylov subspace

Jacobian-free methods

\begin{abstract}
A novel time-stepping shift-invert algorithm for linear stability analysis of laminar flows in complex geometries is presented. This method, based on a Krylov subspace iteration, enables the solution of complex non-symmetric eigenvalue problems in a matrix-free framework. Validations and comparisons to the classical exponential method have been performed in three different cases: (i) stenotic flow, (ii) backward-facing step and (iii) lid-driven swirling flow. Results show that this new approach speeds up the required Krylov subspace iterations and has the capability of converging to specific parts of the global spectrum. It is shown that, although the exponential method remains the method of choice if leading eigenvalues are sought, the performance of the present method could be dramatically improved with the use of a preconditioner. In addition, as opposed to other methods, this strategy can be directly applied to any time-stepper, regardless of the temporal or spatial discretization of the latter.
\end{abstract}

\section{Introduction}

Modal linear stability analysis of a flow, either focusing on the eigenspectrum of the flow or examining the short-time perturbation development, can provide insight into the underlying physical mechanisms of the transition process from a stable steady or time-periodic laminar state to a transitional and turbulent flow state. In order to study this problem, numerical methods based on either matrix-forming $[16,17]$ or matrix-free methods $[7,11,9]$ for flow stability analysis are used. Both matrix-free and matrixforming strategies are based on Krylov subspace methods, such as the Arnoldi iteration, which delivers a part of the eigenspectrum focusing on the dominant or largest modulus eigenvalues. Therefore, a spectrum transformation needs to be carried out in order to recover the least-stable or leading eigenvalues, which responsible for the flow instability. For this, shift-invert transformations are employed in matrix-forming approaches while exponential timestepping strategies are used in matrix-free frameworks. In terms of computational memory required, the latter framework presents clear advantages against approaches in which the matrix is formed when the objective is to study a small number of leading eigenvalues [20].

\footnotetext{
* Corresponding author.

E-mail address: francisco.gomez-carrasco@monash.edu.au (F. Gómez).
}

However, these classical time-stepping matrix-free [2] procedures can be slow and computationally expensive in terms of CPU time [22] when eigenvalues close to the imaginary axis need to be studied. In order to accelerate the procedure to obtain such eigenvalues, an analogous technique to the shift-and-invert strategy employed in the approach in which the matrix is formed can be applied to the time-stepping methods; this idea was first proposed by Goldhirsch et al. [8] in a local instability analysis context and more recently by Tuckerman [22] for the case of bifurcation analysis using inverse matrix-free strategies. The latter proposed using the inverse of the Jacobian in order to obtain the eigenvalues close to the imaginary axis. The action of the inverse Jacobian could be obtained by an iterative procedure such as the Bi-Conjugate Gradient Stabilized algorithm (Bi-CGSTAB) [23]. While the evaluation on the inverse of the Jacobian can be carried out in an efficient way in a matrix-forming context by means of a full $L U$ decomposition, the iterative algorithm could have a slow convergence or not even guarantee convergence in a matrix-free framework. However, a preconditioner based on the Stokes operator can be used to accelerate this iterative procedure. Such preconditioner cannot be directly applied to any time-stepping in a generic way, as shown by Mack and Schmid [14] who successfully resolved this issue for compressible flows by using a Caley transformation [15] applying a low-order inverse Jacobian as an explicit preconditioner matrix.

This paper discusses a new generic methodology to speed up the procedure to obtain leading eigenvalues by means of Krylov 


\section{Nomenclature}

U Base flow

u' Perturbation

Re Reynolds number

$N \quad$ Number of Arnoldi iterations

$N_{p} \quad$ Polynomial order employed in the simulation

$\Delta t \quad$ Integration time

$d t \quad$ Time-step employed in the simulation $\gamma \quad$ Complex eigenvalue

$\sigma \quad$ Shift parameter

tol $_{\text {Arnoldi }}$ Minimum tolerance in Arnoldi method

$N_{\text {Arnoldi }}$ Maximum number of Arnoldi iterations

tol $_{\text {Bi-CGSTAB }}$ Minimum tolerance in Bi-CGSTAB

$N_{B i-C G S T A B}$ Maximum number of Bi-CGSTAB iterations subspace iteration that allows access to specific parts of the linear global eigenspectrum. As will be explained in detail, this methodology is based on a shift transformation with the addition of the application of the inverse Jacobian matrix obtained by timestepping. After discussion of the theory in Section 2, the shiftinvert algorithm for real and complex shifts is presented in Section 3. Results obtained by exponential and shift-invert strategy are presented in Section 4 for three different problems; (i) stenotic flow, (ii) backward-facing step and (iii) lid-driven swirling flow. Finally, conclusions are presented in Section 5.

\section{Theory}

\subsection{General equations}

A time-stepping scheme is used in this work in order to perform the stability study. This method is based on the integration of the incompressible Navier-Stokes equations,

$\nabla \cdot \mathbf{u}=0$

$\partial_{t} \mathbf{u}=\mathbf{A} \mathbf{u}-\nabla p+v \nabla^{2} \mathbf{u}$,

where $\mathbf{A}=-\frac{1}{2}[\mathbf{u} \cdot \nabla \mathbf{u}+\nabla \cdot \mathbf{u u}]$ are the non-linear advection terms, $p$ is the kinematic pressure and $v$ is the kinematic viscosity. The numerical techniques used to integrate this system in the present work were described by Tuckerman and Barkley [22] and Blackburn [4]. It should be re-iterated here that any flow solver, incompressible [4] or compressible, may be used in this context. In this way, the Navier-Stokes equations can be written in a more compact form,

$\partial_{t} \mathbf{u}=\mathbf{N u}+\mathbf{L u}$,

where the pressure term is solved by a Poisson problem in which the condition of divergence-free velocity field is considered, and $\mathbf{N}=-\left(\mathbf{I}-\nabla \nabla^{-2} \nabla \cdot\right) \mathbf{A}$. The numerical solution of the previous system can be expressed symbolically as follows,

$\mathbf{u}(t+\Delta t)=N S_{\Delta t}[\mathbf{u}(t)]$,

where $\Delta t$ is an integration time that can be different to the timestep $d t$ used in the time integration. The specific form of the nonlinear operator $N S_{\Delta t}$ depends on the temporal scheme used to solve the system. For the explicit-implicit Euler time-stepping this operator can be written as follows: $N S_{\Delta t} \approx(\mathbf{I}-\Delta t \mathbf{L})^{-1}(\mathbf{I}+\Delta t \mathbf{N})$. For simplicity this numerical scheme will be considered throughout this paper, although the procedure described can be extended to other temporal-integration schemes.

\subsection{Stability analysis}

The stability analysis studies the evolution of a small perturbation $\mathbf{u}^{\prime}$ superposed at small amplitude, $\epsilon \ll 1$, upon an $O(1)$ steady base flow, $\mathbf{U}$. Although the generalization of the method to analyze time-periodic flows is straightforward, it will be described as applied only to steady flows for simplicity. Substituting the total velocity field, $\mathbf{u}=\mathbf{U}+\epsilon \mathbf{u}^{\prime}$ in Eqs. (2), assuming that $\mathbf{U}$ is a solution of these equations and linearizing the resulting system it is obtained the following Linearized Navier-Stokes Equations (LNSE),

$\partial_{t} \mathbf{u}^{\prime}=\partial_{U} \mathbf{N} \mathbf{u}^{\prime}+\mathbf{L} \mathbf{u}^{\prime}:=L N S_{\Delta t} \mathbf{u}^{\prime}$,

where $\partial_{U} \mathbf{N}$ is the Jacobian of $\mathbf{N}$ around the base flow. Since this operator is linear, it can be expressed as

$L N S_{\Delta t}=\exp [\Delta t(\partial U \mathbf{N}+\mathbf{L})]$

Assuming an exponential time evolution of the perturbation (modal analysis), the system (4) can be converted into an eigenvalue problem defined as follows:

$\left(\partial_{U} \mathbf{N}+\mathbf{L}\right) \mathbf{u}^{\prime}=\gamma \mathbf{u}^{\prime}$,

where $\gamma=\chi+i \psi$ is a complex eigenvalue. The real part represents the growth or damping rate of the perturbation and the complex part is its frequency. A magnitude-angle notation of the complex eigenvalues

$\gamma=\left(\chi^{2}+\psi^{2}\right)^{\frac{1}{2}} \exp \left[i \arctan \left(\frac{\psi}{\chi}\right)\right]$

will be employed through the results in Section 4 for consistency with the exponential transformations.

This problem can be re-written in a more convenient way in order for most unstable eigenvalues to be obtained. To this end, the following (exponential) transformation is used

$\exp \left[\wedge t\left(\partial_{U} \mathbf{N}+\mathbf{L}\right)\right] \mathbf{u}^{\prime}=\Gamma \mathbf{u}^{\prime}$,

where the eigenvalues $\Gamma$ are equal to $\exp (\Delta t \gamma)$ and the eigenvectors remain unchanged with respect to those of (4). Note that the LHS of the previous equations is equal to $L N S_{\triangle t} \mathbf{u}^{\prime}$.

\subsection{Shift-invert strategy}

The exponential transformation, (8), shifts large negative eigenvalues to zero and leading eigenvalues to infinity. However, an issue arises when it is required to access specific parts of the spectrum, for example those eigenvalues with small real and large imaginary parts responsible of Hopf type bifurcations, which do not shift to infinity with this transformation. Therefore, an alternative strategy must be considered in order to obtain these eigenvalues. In designing such an alternative, any function that transforms the original eigenvalue problem (4) must meet three requirements: first, the eigenvectors must remain unchanged, second, the leading eigenvalues of (4) should be dominant eigenvalues of the new eigenvalue problem and third, the conjugate complex pair of eigenvalues defined near the imaginary axis must be separated from the rest of eigenvalues shifting to zero. The exponential shift-invert transformation, defined by

$\left(\exp \left[\Delta t\left(\partial_{U} \mathbf{N}+\mathbf{L}\right)\right]-\sigma \mathbf{I}\right)^{-1} \mathbf{u}^{\prime}=\Gamma \mathbf{u}^{\prime}$,

meets these objectives, where now 


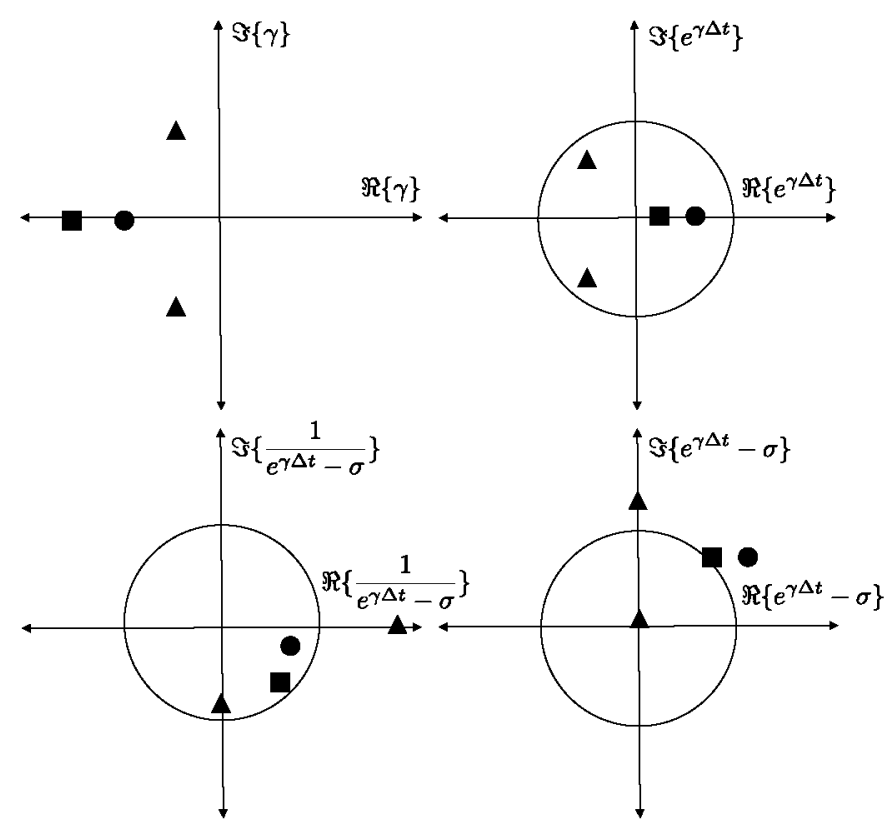

Fig. 1. Dominant eigenvalues as function of the eigenvalue problem considered. Unit circle is shown in the transformed spectra. The shifted eigenvalue (pair of complex eigenvalues represented by a triangle) on the upper left spectrum becomes the dominant eigenvalue by using the shift-invert transformation on the lower left spectrum. Legend: Upper left: Original spectrum. Upper right: Exponential transformation of the spectrum. Lower right: Shift of the exponential transformation of the spectrum. Lower left: Exponential shift-invert transformation of the spectrum.

$\Gamma=\frac{1}{e^{\Delta t \gamma}-\sigma}$

and $\sigma \in \mathbb{R}$. Then, taking $\sigma=1$, eigenvalues of (6) close to zero are mapped to unity by the exponential application, to zero by subtracting $\mathbf{I}$, and to $\infty$ by the inversion. In the general case, eigenvalues of $(6)$ closed to $\log (\sigma)$ are separated from the rest being the dominant eigenvalues. This shift is valid only for real $\sigma$ since the exponential is a real operator. For complex shifts, the following expression can be considered during each Arnoldi iteration,

$$
\begin{array}{cc}
\left(\begin{array}{cc}
\exp \left[\Delta t\left(\partial_{U} \mathbf{N}+\mathbf{L}\right)\right]-\sigma_{r} \mathbf{I} & \sigma_{i} \mathbf{I} \\
-\sigma_{i} \mathbf{I} & \exp \left[\Delta t\left(\partial_{U} \mathbf{N}+\mathbf{L}\right)\right]-\sigma_{r} \mathbf{I}
\end{array}\right) \\
\cdot\left(\begin{array}{l}
\mathbf{u}_{r} \\
\mathbf{u}_{i}
\end{array}\right)=\Gamma\left(\begin{array}{l}
\mathbf{u}_{r} \\
\mathbf{u}_{i}
\end{array}\right)
\end{array}
$$

where $\sigma_{r}=\Re\{\sigma\}$ and $\sigma_{i}=\Im\{\sigma\}$. In this case the leading dimension of the matrix is twice that of the real case, leading to a proportional increase in computational effort when time-stepping is used.

An example of this approach is schematically shown in Fig. 1, in which the original spectrum is defined in the upper left part. In this figure, the most unstable eigenvalue, which corresponds to a pair of complex eigenvalues that wants to be recovered, is represented by a triangle and the dominant eigenvalue is represented by a square. A solid circle represents the second least stable eigenvalue. The exponential transformation of the spectrum is shown in the upper right of Fig. 1. In this transformation, the eigenvalue represented by the circle becomes the dominant eigenvalue and the sought triangle eigenvalue is moved far away from it, therefore it can be hardly recovered with the Arnoldi method. It has to be noticed that the dominant eigenvalue in the original spectrum (squared) is moved to the origin. A complex shift is applied in the spectrum shown in the lower right of Fig. 1, moving the sought eigenvalue to the origin. Finally, an inversion is applied in the spectrum shown in the lower left of Fig. 1, and the eigenvalue represented by the triangle becomes the dominant. This transformed

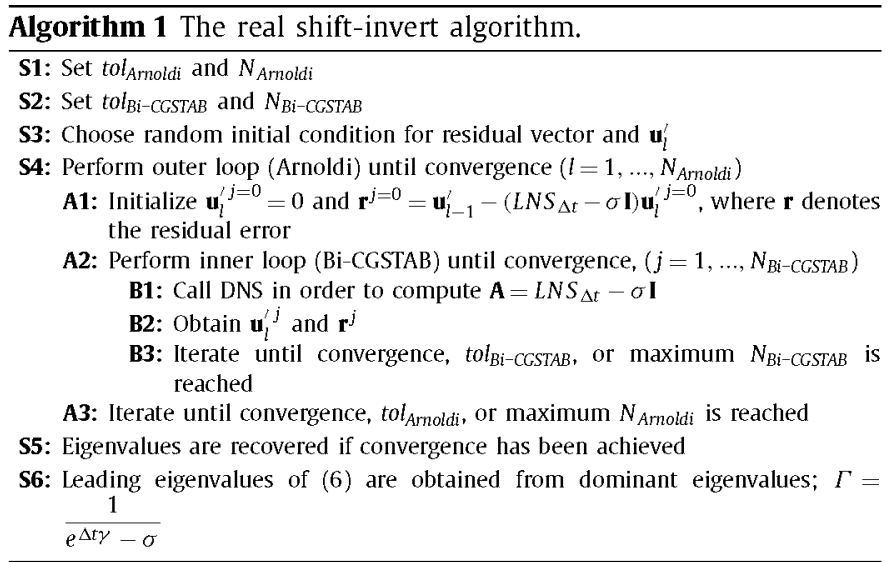

leading eigenvalue can be now easily recovered by applying the Arnoldi algorithm.

\section{Numerical method}

\subsection{The shift-invert algorithm}

The Arnoldi iteration [18] scheme can be used with a real shift in order to obtain the dominant eigenvalues of (9), which are the leading eigenvalues of (6). Then, a sequence of $k$ vectors, $\mathbf{u}_{0}^{\prime}, \mathbf{u}_{1}^{\prime}$, $\mathbf{u}_{2}^{\prime}, \ldots \mathbf{u}_{k-1}^{\prime}$ must be generated in the Arnoldi process from an initial perturbation, $\mathbf{u}_{0}^{\prime}$, for which $\mathbf{u}_{l}^{\prime}=\left(L N S_{\Delta t}-\sigma \mathbf{I}\right)^{-1} \mathbf{u}_{l-1}^{\prime}$ must be provided in each iteration. This implies inversion of the operator, which can be achieved iteratively using a Bi-Conjugate Gradient Stabilized algorithm (Bi-CGSTAG) [23], valid for non-symmetric definite linear systems. In this case, the following operation must be performed in the internal Arnoldi loop, $\left(L N S_{\Delta t}-\sigma \mathbf{I}\right) \mathbf{r}$, where $\mathbf{r}$ is the residual of the method. Therefore, the problem is reduced to solving Eq. (4) by time-stepping a number of times. With respect to the complex shift, the action of the matrix operator defined in (11) involves solving ( $\left.L N S_{\Delta t}-\sigma \mathbf{I}\right) \mathbf{u}_{l-1}^{j}$ separately for the real and the imaginary components. In this case, identical real and complex initial conditions are considered. This scheme is summarized in Algorithm 1.

Reverse communication interfaces for Arnoldi iteration as implemented in ARPACK [12] and the iterative template routine Bi-CGSTAB implemented by Barrett et al. [3] were used in the process described above. This algorithm was implemented in a stability code based on Semtex [4]. Semtex is a spectral direct numerical simulation (DNS) code for the solution of the incompressible Navier-Stokes equations in primitive variables. Standard nodal Gauss-Lobatto-Legendre basis functions are used in two inhomogeneous directions while a Fourier expansion is used in the direction that is assumed homogeneous in three-dimensional problems. More specifically, the domain is divide into blocks and these in quadrilateral obtained by using continuous Galerkin projection from a canonical domain, $[-1,1] \times[-1,1]$. The nodes on each direction are obtained from the zeros of Legendre polynomials. These are generalized to two-dimensional problems by using tensor-product interpolants of one-dimensional grids distribution. The order of the discretization is related with the order of the polynomial and hence with the number of zeros. This imposes restrictions on the definition of Courant-Friedrichs-Lewy (CFL) number, i.e., more restrictive CFL condition implies works with high polynomial order. Finally time integration employs a backwardstime differencing scheme described by Karniadakis et al. [10]. This code has been validated and used in a number of works, see $[4,5]$. A representative selection of these problems is employed as validation cases in Section 4. 
Table 1

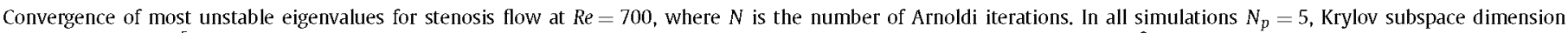
$=8$, tol $l_{\text {Arnoldi }}=10^{-5}, N_{\text {Arnoldi }}=200$. Case $a$ : Exponential method, Case $b$ : Real shift-invert method for $\sigma=0$, tol $l_{B i-C G S T A B}=10^{-3}$ and $N_{B i-C G S T A B}=300 . a \cdot b(c)=a \cdot b \times 10^{c}$.

\begin{tabular}{|c|c|c|c|c|c|}
\hline Cases & Magnitude & Angle & Growth rate & Frequency & $N$ \\
\hline $\mathrm{a}$ & $9.9723(-01)$ & 0.0000 & $-3.7011(-03)$ & 0.0000 & 76 \\
\hline b & $9.9737(-01)$ & 0.0000 & $-3.5113(-03)$ & 0.0000 & 8 \\
\hline
\end{tabular}

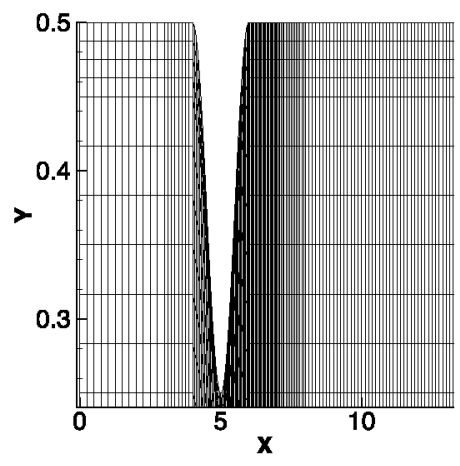

(a) Stenosis

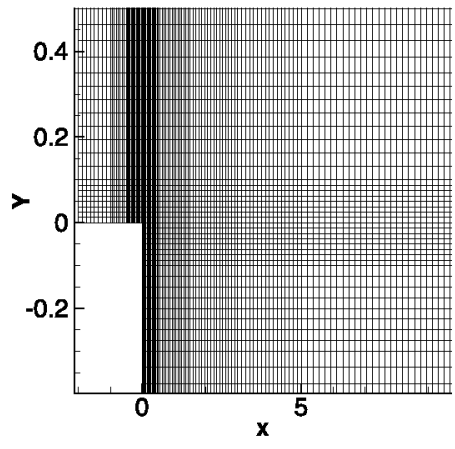

(b) Backward-facing step

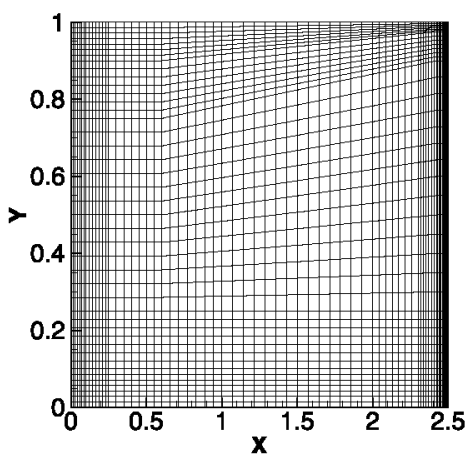

(c) Swirling flow 2D

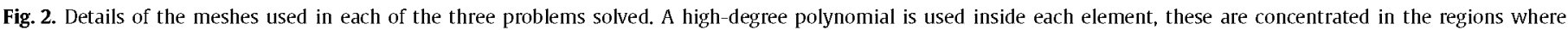
high flow variations are expected.

\subsection{Improving the inversion convergence}

Conjugate gradient iterative methods for non-symmetric definite systems may converge slowly, requiring a large number of iterations when the condition number is high. This is what happens from the spatial discretization of the Navier-Stokes equations, especially for three-dimensional problems where, in addition, the size of the matrices, $L N S_{\Delta t}$, is large. In this case even a moderately large condition number of the operator has an adverse influence on the overall rate of convergence of the iterative method. Preconditioning techniques help improve the convergence of the stability problem, see Knoll and Keyes [11] for a recent overview. The origin of the large condition number is the wide range of eigenvalues of $\mathbf{L}$ and for this reason the Stokes preconditioner $\mathbf{P}=\Delta t(I-\Delta t \mathbf{L})^{-1}$ is often used, see Tuckerman and Barkley [22]. This preconditioner has the disadvantage that cannot be applied directly to a real/complex shift-invert time-stepping and a new preconditioner must be used for the problem at hand. Moreover, the process proposed by these authors [22] is not formally a time-stepping integration because they use only the Stokes operator without pressure and convective terms, and where the time step does not match the CFL condition. Other preconditioning methods are based on the forming and inversion of a low order Jacobian matrix, as proposed by Mack and Schmid [14], but it requires the forming, storing and inversion of a low order Jacobian. In the present shift-invert methodology a general approach based on implicit preconditioning, in the sense of improving the condition number of the matrix to invert without explicitly forming a preconditioner matrix, is applied by means of the time-stepping. This means that the exponential Jacobian matrix is inverted instead of the Jacobian matrix. This preconditioning is only strictly valid for large integration times because the exponential of the Jacobian matrix of the Navier-Stokes equation is not bounded by the exponential of the eigenvalues at short times because its non-normality [6]. However, it will be shown that the employed integrations times are large enough and that the increments in this integration time significantly improve the number of required Bi-CGSTAB iterations for the inversion of the exponential of the Jacobian matrix

\section{Results}

\subsection{Real shift-invert}

Three different problems have been considered in this section in order to validate the present exponential shift-invert method described above: (i) stenosis flow [19], (ii) backward-facing step flow [1] and (iii) lid-driven swirling flow [13]. In all cases, the base solution was obtained using Newton-Raphson iteration started from a numerical solution previously generated with Semtex, see Blackburn [4] for details.

\subsubsection{The stenotic flow: real shift-invert}

Linear stability around the steady stenosis flow at $R e=500$ and $R e=700$ is considered in this section. Fig. 2(a) shows the element mesh and geometry of this case. For these simulations a polynomial order $N_{p}=5$ and $N_{p}=7$ were considered in order to expand flow variables within each element. A low value of $N_{p}=5$ was sufficiently accurate for this study and at the same time permits fast simulations. The Krylov subspace dimension, the maximum number of iterations and the tolerance were taken equal to 8, 200 and $10^{-5}$, respectively.

As seen in Table 1 and Fig. 3, there is a very good agreement between the results obtained with the exponential method (Eq. (8)) and the real shift invert method (Eq. (9)). The most unstable modes obtained using the two strategies agree up to the third decimal place. Different tolerances considered delivered converged solutions in all cases, see Table 2 . As it can be seen, the maximum number of iterations was not achieved in any case, which is a requisite for an accurate solution. It is also remarkable the number of iterations carried out in the internal loop is independent of the Arnoldi tolerance at convergence. Likewise, it can be seen that the number of Arnoldi iterations is drastically reduced from 76 to 8 when the shift-invert method is used in place of the direct method. This however does not imply a reduction in the computational cost, due to the high number of iterations required to invert the matrix on each Arnoldi iteration. Table 2 summarizes these BiCGSTAB iteration results.

In order to evaluate the shifting capability of the method, a value of $\sigma=0.1$ was employed to extract non-leading eigenvalues from the spectrum. Results of these runs at different Arnoldi 


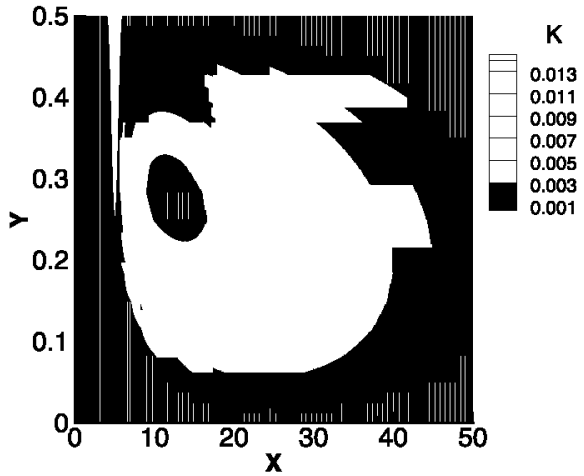

(a)

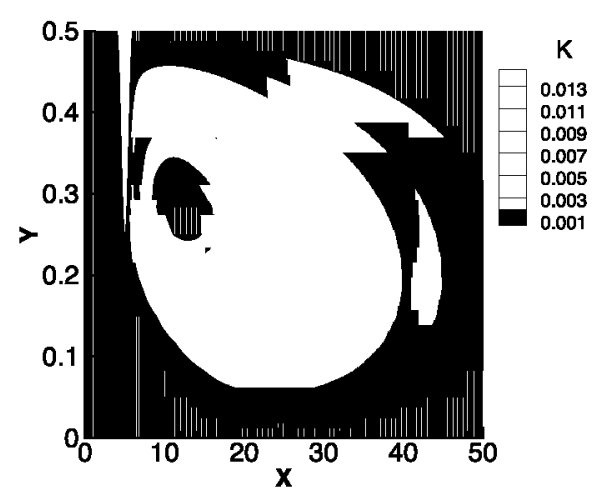

(b)

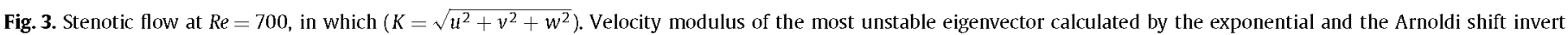
strategy with shift equal to 1. Left: Exponential method. Right: Real shift-invert method.

Table 2

Number of iterations carried out by the Bi-CGSTAB algorithm for the stenosis flow problem at $R e=700$ on each Arnoldi iteration employing a real shift-invert method with $\sigma=0$, Krylov subspace dimension $=8$, tol $l_{\text {Anoldi }}=10^{-5}, N_{\text {Anoldi }}=100$. Case $a$ : Magnitude $=9.9737(-01)$, Growth Rate $=-3.5111(-03)$, tol $_{B i-C G S T A B}=10^{-3}$ and $N_{B i-C G S T A B}=300$. Case b: Magnitude $=9.9737(-01)$, Growth rate $=-3.5113(-03)$, to $l_{B i-C G S T A B}=10^{-4}$ and $N_{B i-C G S T A B}=300 . a \cdot b(c)=a . b \times 10^{c}$.

\begin{tabular}{llc}
\hline Arnoldi Iteration & Case a & Case b \\
\hline 1 & 62 & 73 \\
2 & 43 & 50 \\
3 & 44 & 51 \\
4 & 53 & 53 \\
5 & 66 & 67 \\
6 & 69 & 95 \\
7 & 78 & 101 \\
8 & 80 & 109 \\
\hline
\end{tabular}

Table 3

Number of iterations carried out by the real shift-invert algorithm for the stenosis flow problem at $R e=500$ for different tolerances with $t l_{A \text { rnoldi }}=t_{\text {Bi-CGSTAB }}$ and $\Delta t=6$. Real shift-invert method for $\sigma=0.1$ and Krylov subspace dimension $=8$.

\begin{tabular}{llll}
\hline tol $l_{\text {Bi-CGSTAB }}$ & $10^{-3}$ & $10^{-4}$ & $10^{-5}$ \\
\hline$N_{\text {Annoldi }}$ & 13 & 13 & 13 \\
$N_{\text {Bi-CGSTAB }}$ & 216 & 277 & 312 \\
Growth rate & -0.43269 & -0.43269 & -0.43270 \\
Frequency & 0.037742 & 0.037742 & 0.037748 \\
\hline
\end{tabular}

and Bi-CGSTAB tolerance can be seen in Table 3. The recovery of this eigenvalue has not been possible by using the exponential method at the same resolution and tolerance for any number of iterations or Krylov subspace dimension. In addition, it can be observed in Table 3 that increases in accuracy barely change the Arnoldi iterations required, as it was noticed before. On the other hand, the total number of Bi-CGSTAB iterations increases with the tolerance.

Regarding the effect of the integration time, Table 4 presents the effect of the increase in integration time $\Delta t$ on the total number of Bi-CGSTAB iterations. It can be appreciated that the total number of iterations are reduced as the integration time increases.

\subsubsection{Backward-facing step: real and complex shift-invert}

With increasing $R e$ steady two-dimensional laminar separated backward-facing step flow with a 2:1 longitudinal-to-transversal aspect ratio first becomes unstable to a steady 3D bifurcation at critical Reynolds number about 750, as discovered by Barkley et al. [1], essentially following the same modal scenario predicted by Theofilis et al. [21] in the adverse-pressure-gradient laminar separation bubble flow on a flat plate. The mesh and geometry are represented in Fig. 2(b).
Table 4

Number of iterations carried out by the Bi-CGSTAB algorithm on each Arnoldi iteration for the stenosis flow problem at $R e=500$ and at different integration times $\Delta t$. Real shift-invert method for $\sigma=0$, Krylov subspace dimension $=8$, tol $_{\text {Annoldi }}=$ $10^{-3}, N_{\text {Arnoldi }}=100$, to $_{B \mathrm{~B}-C G S T A B}=10^{-3}$. Magnitude $=9.7378(-01)$, Growth rate $=$ $-5.3146(-02) . a . b(c)=a . b \times 10^{c}$.

\begin{tabular}{lccc}
\hline Arnoldi iteration & $\Delta t=1$ & $\Delta t=2$ & $\Delta t=4$ \\
\hline 1 & 20 & 11 & 5 \\
2 & 22 & 9 & 4 \\
3 & 16 & 8 & 3 \\
4 & 15 & 7 & 4 \\
5 & 18 & 9 & 4 \\
6 & 26 & 10 & 5 \\
7 & 21 & 10 & 4 \\
8 & 28 & 10 & 4 \\
$N_{B i-C G S T A B} \cdot \Delta t$ & 166 & 148 & 132 \\
\hline
\end{tabular}

The most unstable eigenmodes for this configuration have been obtained using the exponential method with Krylov subspace dimension equal to 25 , tol Arnoldi $=10^{-6}$ and maximum number of iterations $N_{\text {Arnoldi }}=500$. Three validation tests are considered in Table 5. The first corresponds to the solution of the problem when the direct method is used, case a, the others are obtained by using the shift-invert method with different resolutions used in the $\mathrm{Bi}$-CGSTAB algorithm, cases $\mathrm{b}$ and $\mathrm{c}$. As it can be seen in case $\mathrm{b}$ of Table 5, the most unstable mode obtained using the shift-invert method and the result obtained using the exponential method agree up to the sixth decimal place. This agreement can also be seen in the eigenvectors obtained with either of the two methods, see Fig. 4.

A value of $\sigma=0$ was considered in the third test case $\mathrm{c}$ in order to validate if the real shift-invert method can converge to the leading eigenvalue when the shift parameter $\sigma$ is taken far away from it, in the absence of other eigenvalues in the real axis. This exercise also leads to the same level of agreement between results of the shift-invert and the exponential methods although, as expected, convergence is worse with respect to the case $b$.

\subsubsection{Two-dimensional swirling flow: complex shift-invert}

In the third problem analyzed, the steady flow in a twodimensional swirling flow has a number of axisymmetric modes, as described by Lopez et al. [13]. At $R e=4000$ a Hopf bifurcation to periodic axisymmetric flow at intermediate aspects ratios ( $\Lambda \approx 2.5$ ) has been identified by these authors. Again, mesh and geometry of the base flow are shown in Fig. 2(c).

The most unstable modes using the exponential method, Krylov subspace dimension equal to 25 , tolerance used on Arnoldi iterations equal to $10^{-6}$ and maximum number of iterations equal to 


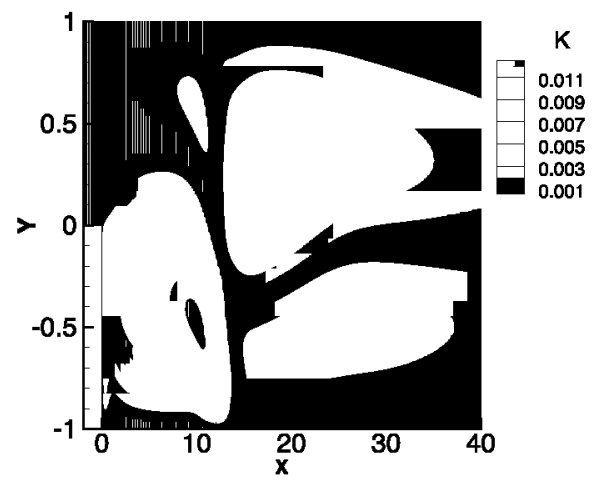

(a)

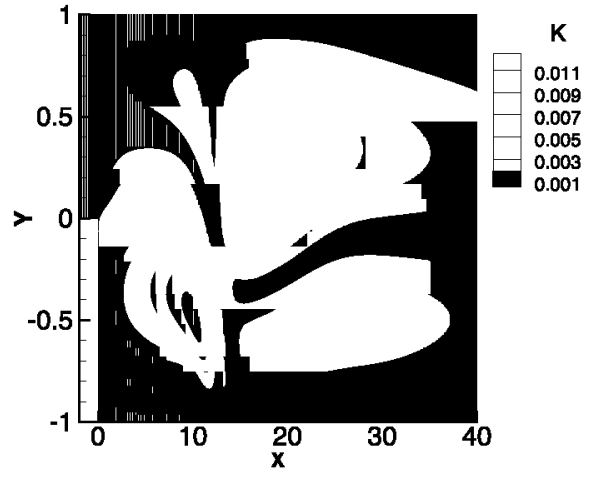

(b)

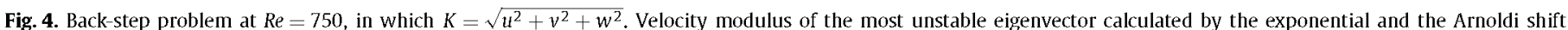
invert strategy with shift equal to 1. Left: Exponential method. Right: Real shift-invert method. See results of Table 5.

Table 5

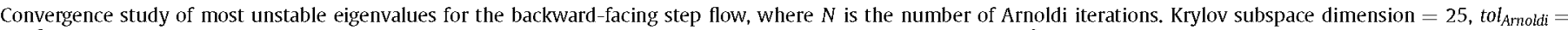

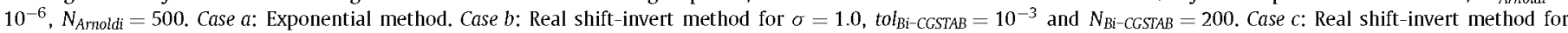
$\sigma=0.0$, to $_{B i-C G S T A B}=10^{-4}$ and $N_{B i-C G S T A B}=300 . a \cdot b(c)=a . b \times 10^{c}$.

\begin{tabular}{|c|c|c|c|c|c|}
\hline Cases & Magnitude & Angle & Growth rate & Frequency & $N$ \\
\hline$a$ & 1.0009 & 0.0000 & $4.2583(-04)$ & 0.0000 & 330 \\
\hline b & 1.0009 & 0.0000 & $4.2579(-04)$ & 0.0000 & 25 \\
\hline c & 1.0008 & 0.0000 & $4.0127(-04)$ & 0.0000 & 25 \\
\hline
\end{tabular}

Table 6

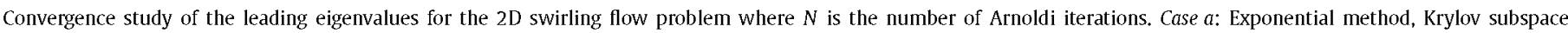

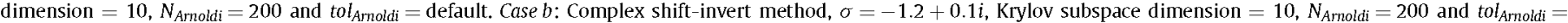
default tol $l_{B i-C G S T A B}=10^{-4}$ and $N_{B i-C G S T A B}=300$. $a \cdot b(c)=a . b \times 10^{c}$.

\begin{tabular}{|c|c|c|c|c|c|c|}
\hline Cases & Eig. & Magnitude & Angle & Growth rate & Frequency & $N$ \\
\hline \multirow[t]{2}{*}{$a$} & 0 & 1.1831 & 3.0637 & $1.2178(-02)$ & $2.2185(-01)$ & 124 \\
\hline & 1 & 1.1831 & -3.0637 & $1.2178(-02)$ & $-2.2185(-01)$ & \\
\hline \multirow[t]{2}{*}{ b } & 0 & 1.1831 & 3.0637 & $1.2178(-02)$ & $2.2185(-01)$ & 10 \\
\hline & 1 & 1.1831 & -3.0637 & $1.2178(-02)$ & $-2.2185(-01)$ & \\
\hline
\end{tabular}

Table 7

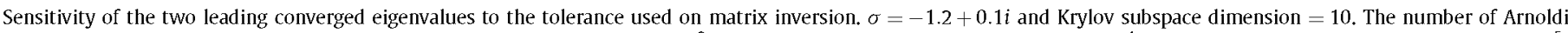

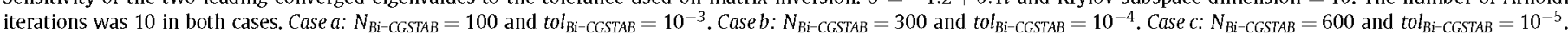
$a . b(c)=a . b \times 10^{c}$

\begin{tabular}{|c|c|c|c|c|c|}
\hline Cases & Eig. & Magnitude & Angle & Growth rate & Frequency \\
\hline \multirow[t]{2}{*}{ a } & 0 & 1.2110 & 3.0735 & $1.3862(-02)$ & $2.2257(-01)$ \\
\hline & 1 & 1.0995 & 3.1183 & $6.8720(-03)$ & $2.2581(-01)$ \\
\hline \multirow[t]{2}{*}{ b } & 0 & 1.1831 & 3.0637 & $1.2178(-02)$ & $2.2185(-01)$ \\
\hline & 1 & 1.1831 & -3.0637 & $1.2178(-02)$ & $-2.2185(-01)$ \\
\hline \multirow[t]{2}{*}{ c } & 0 & 1.1831 & 3.0637 & $1.2178(-02)$ & $2.2185(-01)$ \\
\hline & 1 & 1.1831 & -3.0637 & $1.2178(-02)$ & $-2.2185(-01)$ \\
\hline
\end{tabular}

500 are summarized in Table 6; a component of the respective eigenvectors is shown in Fig. 5.

Several values of the shift parameter $\sigma$ have been considered in this validation problem. A value of $\sigma$ close to the most unstable mode obtained by the exponential method, $-1.2+0.1 i$, was chosen in the first validation test considered in Table 6. The result obtained by the complex shift-invert method is equal to that obtained using the exponential method for the accuracy considered. On the other hand, a comparison of the eigenvectors delivered by both methods, graphically presented in Figs. 5(a) and 5 (c), shows that the second most unstable eigenvalue obtained by the shift-invert method, Fig. 5(c), corresponds to that of the first mode obtained by the exponential method, Fig. 5(a), while the eigenvector of the most unstable mode obtained by the shiftinvert method corresponds to the second mode delivered by the exponential method. Additionally, a global change of phase was observed between both formulations. However, this effect cannot be seen in the velocity modulus where the phase shift is removed.

Particular attention has been paid to the convergence of eigenvalues during this validation using several combinations of the related parameters, since two iterative processes are involved, the external loop (Arnoldi iteration) and the internal loop (Bi-CGSTAB iteration). The effect of tolerance used on the matrix inversion is summarized in Table 7. The tolerance considered in the first case was too low for convergence of the eigenvalues. However, results based on cases $b$ and $c$ indicate that convergence is achieved.

\section{Conclusions}

A time-stepping solver has been successfully applied to study global instability analysis using a new shift-invert strategy, aiming at the efficient capturing of any eigenvalue of the spectrum. The 


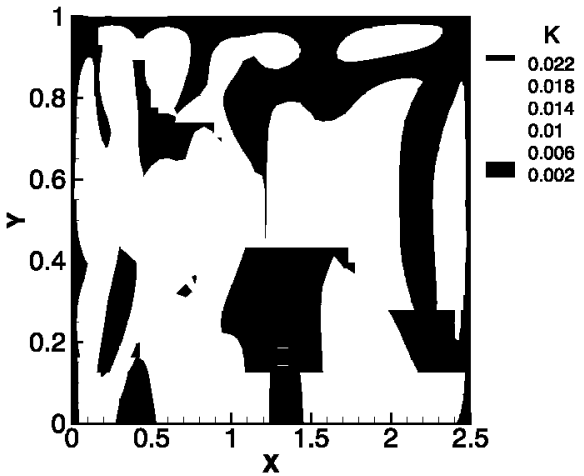

(a) $K$. Eig 1

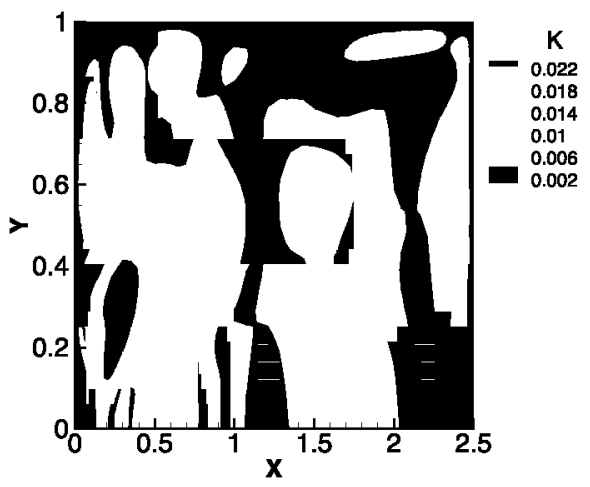

(c) $K$. Eig 2

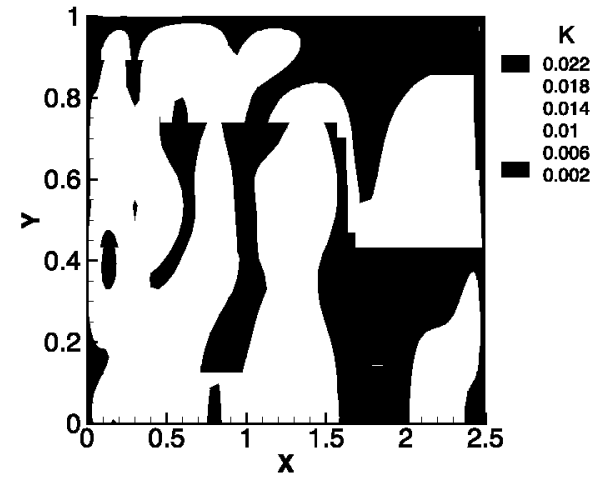

(b) $K$. Eig 2

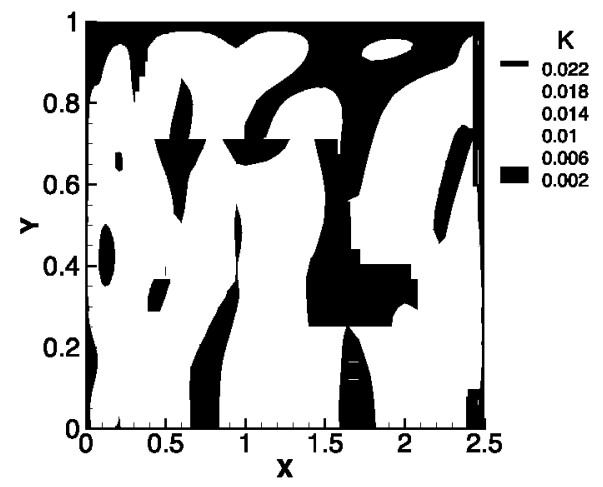

(d) $K$. Eig 1

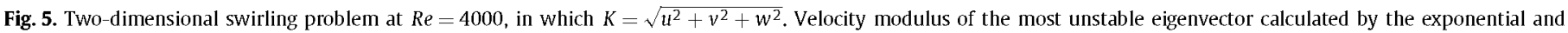
the Arnoldi shift invert strategy with shift equal to $-1.2+0.1 i$. Upper: Exponential method. Lower: Real shift-invert method.

Arnoldi iteration with an embedded Bi-CGSTAB iteration have been employed and the resulting algorithm was shown to dramatically improve the convergence properties of the Arnoldi iterations in all test cases examined, in which real and complex-conjugate pair of eigenvalues where delivered. However, the inversion of the Jacobian matrix required a significant number of $\mathrm{Bi}-\mathrm{CGSTAB}$ iterations in order to converge, leaving the classical exponential method as the method of choice for the recovery of leading eigenvalues. On the other hand, the effect of the preconditioner on the methodology performance has been shown to be critical and the search of a more efficient preconditioner than the employed in this work remains as a future task. Additionally, a strength of this novel method consists in accessing subsets of the spectrum thus this methodology could complement the classical exponential method when a broader access to the eigenspectrum is required. Finally, as opposed to other methods described through the paper, this strategy can be directly applied to any time-stepper, regardless of its temporal or spatial discretization.

\section{Conflict of interest statement}

None declared.

\section{Acknowledgements}

Support of the Spanish Ministry of Science and Innovation through Grant MICINN-TRA2009-13648: "Metodologias computacionales para la predicción de inestabilidades globales hidrodinámicas $y$ aeroacuisticas de flujos complejos" and support of the Marie Curie Grant PIRSES-GA-2009-247651 "FP7-PEOPLE-IRSES: ICOMASEF - Instability and Control of Massively Separated Flows" are gratefully acknowledged.

\section{References}

[1] D. Barkley, M. Grabiela, M. Gomes, R.D. Henderson, Three-dimensional instability in flow over a backward-facing step, J. Fluid Mech. 473 (2002) 167-190.

[2] D. Barkley, H.M. Blackburn, S.J. Sherwin, Direct optimal growth analysis for timesteppers, Int. J. Num. Methods in Fluids 57 (2008) 1435-1458.

[3] R. Barrett, M. Berry, T. Chan, J. Demmel, J. Donato, V.E.J. Dongarra, R. Pozo, C. Romine, H. van den Horst, Templates for the Solutions of Linear Systems: Building Blocks for Iterative Methods, SIAM, Philadelphia, 1993.

[4] H.M. Blackburn, Three-dimensional instability and state selection in an oscillatory axisymmetric swirling flow, Phys. Fluids 14 (11) (2002) 3983-3996.

[5] H.M. Blackburn, R. Henderson, A study of two-dimensional flow past an oscillating cylinder, J. Fluid Mech. 385 (1999) 255-286.

[6] J.-M. Chomaz, Global instabilities in spatially developing flows: non-normality and nonlinearity, Annu. Rev. Fluid Mech. 37 (2005) 357-392.

[7] L. Eriksson, A. Rizzi, Computer-aided analysis of the convergence to steady state of discrete approximations to the Euler equations, J. Comput. Phys. 57 (1985) $90-128$.

[8] I. Goldhirsch, S.A. Orszag, B.K. Maulik, An efficient method for computing leading eigenvalues and eigenvectors of large asymmetric matrices, J. Sci. Comput. 2 (1987) 33-58.

[9] F. Gomez, R. Gomez, V. Theofilis, On three-dimensional global linear instability analysis of flows with standard aerodynamics codes, Aerosp. Sci. Technol. 32 (2014) 223-234.

[10] G. Karniadakis, M. Israeli, S. Orszag, High-order splitting methods for the incompressible Navier-Stokes equations, J. Comput. Phys. 97 (1991) 441-443.

[11] D.A. Knoll, D. Keyes, Jacobian-free Newton-Krylov methods: a survey of approaches and applications, J. Comput. Phys. 193 (2004) 357-397.

[12] R.B. Lehoucq, D.C. Sorensen, C. Yang, Arpack Users Guide: Solution of LargeScale Eigenvalue Problems with Implicitly Restarted Arnoldi Methods, SIAM, Philadelphia, ISBN 978-0898714074, 1998.

[13] J.M. Lopez, F. Marques, J. Sanchez, Oscillatory modes in an enclosed swirling flow, J. Fluid Mech. 439 (2001) 109-129.

[14] C.J. Mack, P.J. Schmid, A preconditioned Krylov technique for global hydrodynamic stability analysis of large-scale compressible flows, J. Comput. Phys. 229 (2010) 541-560.

[15] M. Morzyńksi, F. Thiele, Numerical stability analysis of flow about a cylinder, Z. Angew. Math. Mech. 71 (1991) T424-T428.

[16] R. Natarajan, A. Acrivos, The instability of the steady flow past spheres and disks, J. Fluid Mech. 254 (1993) 323-344. 
[17] P. Paredes, M. Hermanns, S. Le Clainche, V. Theofilis, Order $10^{4}$ speedup in global linear instability analysis using matrix formation, Comput. Methods Appl. Mech. Eng. 253 (2013) 287-304.

[18] Y. Saad, Variations of Arnoldi's method for computing eigenelements of large unsymmetric matrices, Linear Algebra Appl. 34 (1980) 269-295.

[19] S.J. Sherwin, H.M. Blackburn, Three-dimensional instabilities and transition of steady and pulsatile axisymmetric stenotic flows, J. Fluid Mech. 533 (2005) $297-327$.

[20] V. Theofilis, Global linear instability, Annu. Rev. Fluid Mech. 43 (2011) 319-352.
[21] V. Theofilis, S. Hein, U. Dallmann, On the origins of unsteadiness and three dimensionality in a laminar separation bubble, Philos. Trans. R. Soc. Lond. A 358 (2000) 3229-3246.

[22] L. Tuckerman, D. Barkley, Bifurcation analysis for timesteppers, in: E. Doedel, L. Tuckerman (Eds.), Numerical Methods for Bifurcation Problems and Large-Scale Dynamical Systems, Springer, Berlin, 2000, pp. 453-566.

[23] H.A. Van der Vorst, Bi-CGSTAB: a fast and smoothly converging variant of Bi-CG for the solution of nonsymmetric linear systems, SIAM J. Sci. Stat. Comput. 13 (1992) 631-644. 